\title{
A Method to Determine Stress-Dependent Filtration Properties of Fractured Porous Rocks by Laboratory Test Data
}

\author{
Larisa Nazarova ${ }^{1, *}$, Leonid Nazarov ${ }^{1}$, Nikita Golikov ${ }^{2}$ and Alexandr Skulkin ${ }^{1}$ \\ ${ }^{1}$ Chinakal Institute of Mining, Siberian Branch, Russian Academy of Sciences, 630091, Novosibirsk, \\ Russia \\ ${ }^{2}$ Trofimuk Institute of Petroleum Geology and Geophysics, Siberian Branch, Russian Academy of \\ Sciences, 630090, Novosibirsk, Russia
}

\begin{abstract}
Within the dual porosity model the authors develop and theoretically, using synthetic data, substantiate the method for determining mass exchange coefficient and stress-dependent permeability of fractured porous reservoir rocks. The proposed filtration test circuit consists of three sequential measurements of flowrate in a specimen subjected to the varied external stress $\sigma$ at the inlet fluid pressure $P$ : by standard scheme $\left(Q_{0}\right)$ and with plugging of fissures at one $\left(Q_{1}\right)$ and at the other end $\left(Q_{2}\right)$ of the specimen. The model of the experiment is created, and the analytical solution is obtained for the direct problem on steady-state flow: dependences of $Q_{0}, Q_{1}$ and $Q_{2}$ on $\sigma$ and $P$. The input data are synthesized by superimposition of multiplicative noise on the exact solution of the direct problem. The synthesized data are used to derive the inversion relations for calculating the permeabilities $k_{1}$ and $k_{2}$ of fissures and matrix as well as the mass exchange coefficient by $Q_{0}, Q_{1}$ and $Q_{2}$. Using LS method, the dependences $k_{1}(\sigma)$ and $k_{2}(\sigma)$ are reconstructed. The numerical experiments reveal low stability of inversion by input data. Thus, it is necessary to perform a cycle of measurements at the increasing input pressure with subsequent averaging of the results.
\end{abstract}

\section{Introduction}

Justification of opening and mining schemes in hydrocarbon extraction, estimation of well stability, inversion of logging data - this is a far from being complete list of problems solvable using knowledge on poroperm properties of reservoir rocks [1-3]. The source of such information can be laboratory experiments, field tests and integrated geophysical logging $[4,5]$. The obtained data are interpreted in the framework of hydrodynamic models usually selected based on the petrographic analysis of core samples [6].

\footnotetext{
* Corresponding author: lanazarova@ngs.ru
} 
Productive strata in many hydrocarbon reservoirs have porous and fractured structure [7]. For description of fluid flow in such strata, in [8] a dual porosity model is proposed for a medium composed of elements of two types - matrix and fissures (Fig. 1). Each type is provided with a set of the governing parameters (pressure, permeability, porosity, etc.) and the law of mass exchange between the constituent elements. Later on, this approach was generalized to any types of continuum [9-11] by introduction of notion of a representative elementary volume and was successfully applied in studying heat and mass transfer processes in multi-phase systems $[12,13]$, including oil and gas reservoirs [14-17] and coal rock masses [18-22].

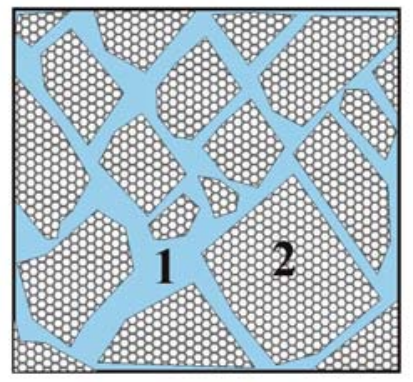

Fig. 1. Structure of fractured porous medium: 1 - fissures; 2 - matrix.

One of the stages in verification of such models is estimation of permeability and porosity of constituent elements, as well as determination of an empirical function $M$ describing mass exchange between matrix and fissures. The objective is reached using data of laboratory [23, 24] and full-scale [25-27] tests, as well as calculated effective parameters of media with regular $[28,29]$ and stochastic $[30,31]$ structure. In $[8,25]$, it is assumed that the function $M$ can only be determined in the transient mode of well operation using the pressure build-up curve.

The laboratory [32-36] and in-situ [37-39] researches prove the essential dependence of rock permeability on stresses. With increasing depth, this effect exerts stronger influence on efficiency of geophysical log data interpretation aimed to identify producing intervals and estimate poroperm properties of reservoir rocks [40, 41].

This paper, within the model of the fractured porous medium [8], develops and theoretically substantiates the method which makes it possible to determine the empirical function of mass exchange between matrix and fissures as well as the dependence of their permeabilities on stresses by the data of stationary filtration tests.

\section{Model of Fractured Porous Medium}

Evolution of hydrodynamic fields in a fractured porous medium is described with the dual porosity model [8] including:

the continuity equations

$$
\left(\varphi_{1} \rho_{1}\right)_{, t}+\nabla \cdot\left(\rho_{1} \mathbf{V}_{1}\right)+M=0, \quad\left(\varphi_{2} \rho_{2}\right)_{, t}+\nabla \cdot\left(\rho_{2} \mathbf{V}_{2}\right)-M=0
$$

Darcy's law

and the state equations

$$
\mathbf{V}_{m}=-k_{m} \nabla p_{m} / \eta
$$

$$
\rho_{m} / \rho_{0}=1+c\left(p_{m}-P_{a}\right), \quad \varphi_{m}=\varphi_{m}^{0}+c_{m}\left(p_{m}-P_{a}\right),
$$

where subscripts 1 and 2 denote values related with fissures and matrix, respectively, and superscript 0 - values of the same parameters under the atmospheric pressure $P_{a} ; \mathbf{V}_{m}$ is the 
fluid flow velocity $(m=1,2) ; \rho_{m}, \varphi_{m}$ and $c_{m}$ are the density, porosity and compressibility of solid substance; $\rho, \eta$ and $c$ are, respectively, the density, viscosity and compressibility of fluid. The laboratory tests $[34,35,42,43]$ show that under low pressures, the permeability dependence on stresses is well approximated by the exponential function

$$
k_{m}=K_{m} \exp \left(-\alpha_{m} \sigma\right)
$$

$K_{m}$ and $\alpha_{m}$ are empirical constants. In case of comparable pressure and stresses, $\sigma$ in (4) should be interpreted as an effective stress $\sigma-p_{m}$. For rocks with porosity of $5-20 \%$, $\alpha_{2} \cong 0.001-0.1 \mathrm{MPa}^{-1}$.

In $[8,43]$ it is proposed to describe intensity of crossflows between matrix and fissures by the linear function

$$
M\left(p_{1}, p_{2}\right)=\rho_{0} \beta\left(p_{2}-p_{1}\right) / \eta
$$

where $\beta$ is the mass exchange coefficient. In $[8,25]$ it is mentioned that $\beta$ depends on the specific surface of matrix rocks.

When fluid, matrix and fissures are the weakly compressible media, system (1)-(3) can be linearized and, with regard to (5), reduced to two equations

$$
\begin{aligned}
& \left(c_{1}+c \varphi_{1}^{0}\right) \eta \frac{\partial p_{1}}{\partial t}=k_{1} \Delta p_{1}-\beta\left(p_{2}-p_{1}\right), \\
& \left(c_{2}+c \varphi_{2}^{0}\right) \eta \frac{\partial p_{2}}{\partial t}=k_{2} \Delta p_{2}+\beta\left(p_{2}-p_{1}\right) .
\end{aligned}
$$

\section{Formulation and Solution of Boundary Problem}

Let a cylindrical specimen (base area $S$, length $l$ ) be subjected to a constant stress $\sigma$ over lateral surface (Fig. 2). We consider a one-dimensional steady-state flow described by the system of equations obtained from (6)

$$
\begin{aligned}
& k_{1} \frac{\partial^{2} p_{1}}{\partial x^{2}}-\beta\left(p_{2}-p_{1}\right)=0 \\
& k_{2} \frac{\partial^{2} p_{2}}{\partial x^{2}}+\beta\left(p_{2}-p_{1}\right)=0
\end{aligned}
$$

where $p_{1}$ and $p_{2}$ are the functions of the coordinate $x$ directed along the specimen axis. The permeability-stress dependence is included in (7) through (4).

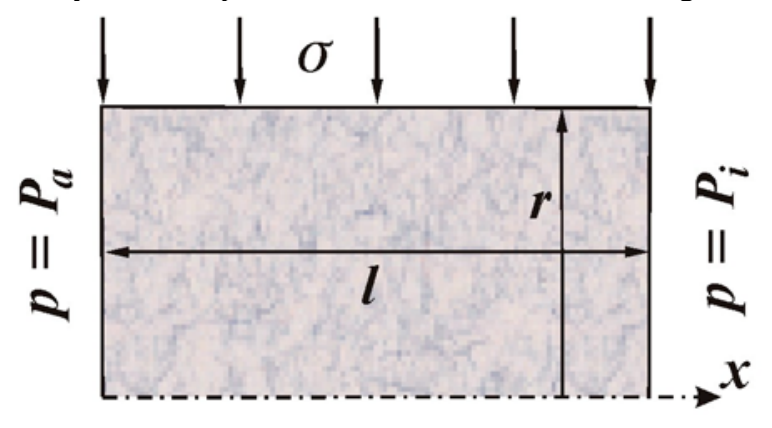

Fig. 2. Scheme of experiment.

As per the study objective, it is required to develop the filtration test pattern such that to find the mass exchange coefficient and constants in empirical dependences (4) of stress- 
dependent permeabilities of matrix and fissures. Determination of the parameters $k_{1}, k_{2}$ and $\beta$ included in (7) needs not less than three 'quantums' of information obtained on the same specimen. The three sequential measurement designs are presented below with relevant boundary conditions for (7).

T0. Standard filtration tests [45]

$$
p_{1}(0)=p_{2}(0)=P_{a}, \quad p_{1}(l)=p_{2}(l)=P_{i},
$$

$P_{i}$ is the inlet pressure (Fig. 2).

T1. On the left end, fissures are plugged with a penetrant, and using a high resolution image (obtained, e.g., with electron microscope), the area $S_{1}$ of these fissures is determined. On the right end $x=l$, the fluid pressure $P_{i}$ is set; on the left end, the atmospheric pressure is assigned

$$
V_{1}(0)=0, p_{2}(0)=P_{a}, \quad p_{1}(l)=p_{2}(l)=P_{i} .
$$

T2. The right end fissures are plugged and then

$$
V_{1}(0)=0, \quad p_{2}(0)=P_{a}, \quad V_{1}(l)=0, \quad p_{2}(l)=P_{i} .
$$

In all tests T0, T1 and T2, the fluid flow rates $Q_{0}, Q_{1}$ and $Q_{2}$ are measured on the left end in the steady-state flow mode.

Omitting cumbersome intermediate calculations, Table 1 presents the final result, namely, the pressure distribution in matrix and fissures, as well as the fluid flowrate at $x=0$.

Table 1. Solving system of equations (7) at the boundary conditions T0, T1 and T2

\begin{tabular}{|c|c|}
\hline T0 & $p_{1}(\xi)=p_{2}(x)=P_{a}+\left(P_{i}-P_{a}\right) \xi$ \\
& $Q_{0}=\frac{k_{1}(\delta+\psi)\left(p_{c}-p_{a}\right) S}{(1+\delta) l \eta}$ \\
\hline T1 & $p_{1}(\xi)=P_{a}+\left(P_{i}-P_{a}\right) \frac{D(\gamma, \xi)+\xi \sin \gamma(1-\xi)}{D(\gamma, 1)}$ \\
& $p_{2}(\xi)=P_{a}+\left(P_{i}-P_{a}\right) \frac{D(\gamma, \xi)-\sin \gamma(1-\xi)}{D(\gamma, 1)}$ \\
T2 & $Q_{1}=\frac{\psi k_{1}\left(p_{c}-p_{a}\right) S}{(1+\delta) l \eta} G(\gamma, \xi)$ \\
& $p_{1}(\xi)=P_{a}+\left(P_{i}-P_{a}\right) \frac{D(\gamma, \xi)+\xi \sin \gamma(1-\xi)}{D(\gamma, 1)}$ \\
& $p_{2}(\xi)=P_{a}+\left(P_{i}-P_{a}\right) \frac{D(\gamma, \xi)-\sin \gamma(1-\xi)}{D(\gamma, 1)}$ \\
& $Q_{2}=\frac{\psi k_{1}\left(p_{c}-p_{a}\right) S}{(1+\delta) l \eta} G(\gamma / 2, \xi)$
\end{tabular}

In (8)-(13) we introduced symbols $\xi=x / l, \quad \psi=k_{2} / k_{1}, \quad \gamma=l \sqrt{\beta(1+\psi) / k_{1}}$, $\delta=S_{1} /\left(S-S_{1}\right), D(\gamma, \xi)=\sin \gamma+\gamma \psi \xi \cos \gamma, G(\gamma, \xi)=\gamma(1+\psi) /(\tan \gamma+\psi \gamma)$.

Figure $3 \mathrm{a}$ depicts the pressure in matrix in the test design T2 at $P_{i}=0.2 \mathrm{MPa}$, $P_{a}=0.1 \mathrm{MPa}, \psi=0.02$ and different values of $\gamma$. The distribution of $p_{2}$ is asymmetrical relative to the middle of the specimen.

Figure $3 \mathrm{~b}$ demonstrates the contour lines of the dimensionless flowrate $G(\gamma, \psi): G$ weakly depends on $\gamma$ at small $\psi$. This is one of the reasons for instable inversion of the experimental results by the input data (paragraph 4 ). 

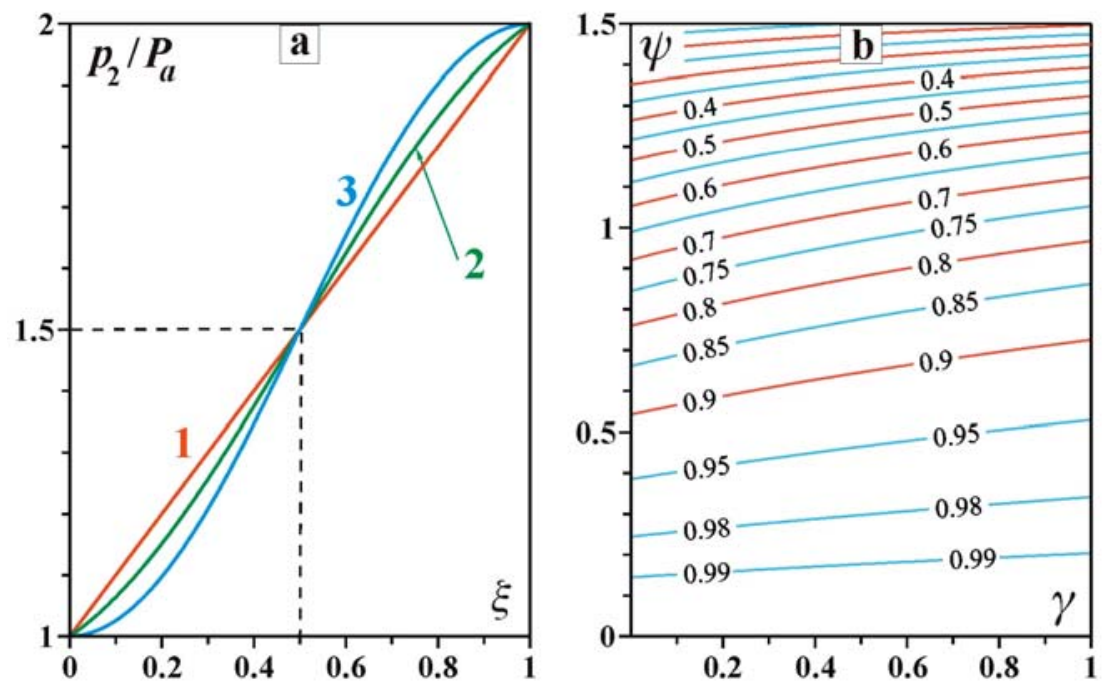

Fig. 3. Pressure distribution in matrix at boundary conditions T2 (a); contour lines of function $G$ (b).

\section{Inversion of Experimental Results}

\subsection{Synthesis of Input Data}

We set some values for the permeabilities $K_{1}=K_{1}^{*}$ and $K_{2}=K_{2}^{*}$, mass exchange coefficient $\beta=\beta^{*}$, as well as the parameters $\alpha_{1}=\alpha_{1}^{*}$ and $\alpha_{2}=\alpha_{2}^{*}$. The specimen has radius $r=0.2 \mathrm{~cm}\left(S=12.57 \mathrm{~cm}^{2}\right)$ and length $l=8 \mathrm{~cm}$; the relative area of fissures on the left end is $\delta=0.001$, the fluid viscosity is $\eta=10^{-5} \mathrm{~Pa} \cdot \mathrm{s}$ (air). For each value of the inlet pressure $P_{i}$ and external stress $\sigma=\sigma_{n}$ in the chosen ranges $\left[P_{\min }, P_{\max }\right]$ and $\left[\sigma_{\min }, \sigma_{\max }\right]$, from formulas (9), (11) and (13), we calculate the flow rates $Q_{s}^{*}(i, n)(s=0,1,2)$ and synthesize input data by superposing the exact values with multiplicative noise

$$
Q_{s}(i, n)=\left[1+\omega_{s}(i, n)\right] Q_{s}^{*}(i, n),
$$

where $\omega_{s}(i, n)$ are random values uniformly distributed in the segment $[-\varepsilon, \varepsilon], \varepsilon$ is a relative error of measurements. Modern flowrate meters have $\varepsilon$ lesser than 0.5-1\% [46].

The numerical experiments involved $K_{1}^{*}=2500 \mathrm{mD}, K_{2}^{*}=125 \mathrm{mD}, \quad \beta^{*}=0.012$, $\alpha_{1}^{*}=0.025 \mathrm{MPa}^{-1}, \quad \alpha_{2}^{*}=0.005 \mathrm{MPa}^{-1}, \quad P_{\min }=0.2 \mathrm{MPa}, \quad P_{\max }=0.7 \mathrm{MPa}, \quad \sigma_{\min }=0$, $\sigma_{\max }=5 \mathrm{MPa}$. Table 2 presents the input data (columns 2, 3 and 4) generated using (14) at $\varepsilon=0.01$.

\subsection{Determination of Poroperm Parameters of Fractured Porous Medium}

Denote $u_{1}=Q_{1}(i, n) / Q_{0}(i, n), u_{2}=Q_{2}(i, n) / Q_{0}(i, n)$, then, dividing (11) and (13) by (9), we obtain

$$
\psi G(\gamma, \psi) /(\delta+\psi)=u_{1}, \quad \psi G(\gamma / 2, \psi) /(\delta+\psi)=u_{2} .
$$


The Macluarin expansion of the tangent at small $\gamma$ reduces (15) to a system of equations with respect to $\gamma$ and $\psi$

$$
\frac{\psi(1+\psi)}{(\delta+\psi)\left(1+\psi+\gamma^{2} / 3\right)}=u_{1}, \quad \frac{\psi(1+\psi)}{(\delta+\psi)\left(1+\psi+\gamma^{2} / 12\right)}=u_{2}
$$

which has an analytical solution (columns 5 and 6, Table 2)

$$
\psi=\frac{3 u_{1} u_{2} \delta}{4 u_{1}-u_{2}-3 u_{1} u_{2}}, \quad \gamma^{2}=\frac{12(1+\psi)\left(u_{2}-u_{1}\right)}{4 u_{1}-u_{2}}
$$

The permeability of fissures at the preset external stress $\sigma_{n}$ and inlet pressure $P_{i}$ is found from (9)

$$
k_{1}=\frac{\eta l(1+\delta) Q_{0}(i, n)}{\left(P_{i}-P_{a}\right)(\delta+\psi) S}
$$

(columns 6), the matrix permeability $k_{2}=\psi k_{1}$ (columns 8) and, finally, the mass exchange coefficient

$$
\beta=\frac{\gamma^{2} k_{1}}{\eta l^{2}(1+\psi)}
$$

(columns 10). For each $\sigma_{n}$, from (4) we find the exact values of the permeabilities $k_{m}^{*}=K_{m}^{*} \exp \left(-\alpha_{m}^{*} \sigma_{n}\right) \quad$ and calculate the relative errors $\lambda_{1}=\left|1-k_{1} / k_{1}^{*}\right|$ and $\lambda_{2}=\left|1-k_{2} / k_{2}^{*}\right|$, as well as $\lambda_{\beta}=\left|1-\beta / \beta^{*}\right|$ (columns 8,10 and 12 , Table 2 ). It turns out that even at the small measurement error of the flowrate, the determination error of $k_{1}$ and $\beta$ can exceed $70 \%$. The cause of such errors lies in the contrast of the permeabilities of matrix and fissures as they can differ by 1-3 orders of magnitude [25]. For better accuracy,

\begin{tabular}{|c|c|c|c|c|c|c|c|c|c|c|c|}
\hline 1 & 2 & 3 & 4 & 5 & 6 & 7 & 8 & 9 & 10 & 11 & 12 \\
\hline $\begin{array}{c}P_{i} \\
M P a\end{array}$ & $\begin{array}{c}Q_{0} \\
l / \mathrm{min}\end{array}$ & $\begin{array}{c}Q_{1} \\
\text { l/min }\end{array}$ & $\begin{array}{c}Q_{2} \\
l / \mathrm{min}\end{array}$ & $\xi$ & $\gamma$ & $\begin{array}{c}k_{1} \\
m D\end{array}$ & $\lambda_{1}, \%$ & $\begin{array}{c}k_{2} \\
m D\end{array}$ & $\lambda_{2}, \%$ & $\beta$ & $\lambda_{\beta}, \%$ \\
\hline 0.20 & 1.03 & 0.99 & 1.01 & 0.1140 & 0.3177 & 946.8 & 19.8 & 107.9 & 0.3 & 1.528 & 27.3 \\
\hline 0.25 & 1.55 & 1.48 & 1.51 & 0.1016 & 0.3026 & 1060.2 & 10.2 & 107.8 & 0.2 & 1.399 & 16.6 \\
\hline 0.30 & 2.05 & 1.98 & 2.01 & 0.0977 & 0.2605 & 1102.1 & 6.7 & 107.7 & 0.1 & 1.040 & 13.3 \\
\hline 0.35 & 2.57 & 2.48 & 2.52 & 0.1155 & 0.2830 & 934.3 & 20.9 & 107.9 & 0.3 & 1.211 & 0.9 \\
\hline 0.40 & 3.09 & 2.97 & 3.03 & 0.1247 & 0.3067 & 866.5 & 26.6 & 108.0 & 0.4 & 1.412 & 17.6 \\
\hline 0.45 & 3.59 & 3.45 & 3.52 & 0.1382 & 0.3215 & 782.4 & 33.7 & 108.1 & 0.5 & 1.535 & 27.9 \\
\hline 0.50 & 4.09 & 3.95 & 4.04 & 0.2254 & 0.3326 & 481.7 & 59.2 & 108.6 & 0.9 & 1.531 & 27.6 \\
\hline 0.55 & 4.65 & 4.44 & 4.52 & 0.0627 & 0.2736 & 1704.5 & 44.3 & 106.9 & 0.7 & 1.176 & 2.0 \\
\hline 0.60 & 5.13 & 4.93 & 5.04 & 0.1443 & 0.3254 & 749.5 & 36.5 & 108.2 & 0.6 & 1.564 & 30.3 \\
\hline 0.65 & 5.67 & 5.42 & 5.52 & 0.0642 & 0.2691 & 1664.7 & 41.0 & 106.9 & 0.6 & 1.137 & 5.3 \\
\hline 0.70 & 6.18 & 5.95 & 6.06 & 0.1096 & 0.2874 & 983.9 & 16.7 & 107.9 & 0.3 & 1.255 & 4.6 \\
\hline & & & & & & 1025.1 & 13.2 & 107.8 & 0.2 & 1.344 & 12.0 \\
\hline
\end{tabular}
it is necessary to measure flow rates at several values of the input pressure $P_{i}$ and to assume the required values as average quantities (last line in Table 2).

Table 2. Input data generated at $\sigma=3 \mathrm{MPa}$ and the virtual experimental results.

\subsection{Determination of Stress-Dependent Permeabilities of Matrix and Fissures}

For the model parameters accepted in 4.2 but at $\varepsilon=0.02$, using (14), we synthesize input data for $P_{i}=0.2,0.25, \ldots, 0.7 \mathrm{MPa}$ and $\sigma_{n}=0,1, \ldots, 5 \mathrm{MPa}$, perform inversion and find permeabilities for fissures, $k_{1}(i, n)$, and matrix, $k_{2}(i, n)$ (columns 2-7, Tables 3 and 4). 
Table 3. Virtual experimental results on reconstruction of the dependence between the fissure permeability $k_{1}$ and stresses.

\begin{tabular}{|c|c|c|c|c|c|c|c|c|c|c|c|}
\hline 1 & 2 & 3 & 4 & 5 & 6 & 7 & 8 & 9 & 10 & 11 & 12 \\
\hline \multirow{2}{*}{$\begin{array}{c}P_{i} \\
M P a\end{array}$} & \multicolumn{6}{|c|}{$\sigma_{n}, M P a$} & \multirow{2}{*}{$\begin{array}{c}K_{1} \\
m D\end{array}$} & \multirow{2}{*}{$\begin{array}{l}\theta_{1} \\
\%\end{array}$} & \multirow{2}{*}{$\begin{array}{c}\alpha_{1} \\
M P a^{-1}\end{array}$} & \multirow{2}{*}{$\begin{array}{l}\theta_{\alpha} \\
\%\end{array}$} & \multirow{2}{*}{$\begin{array}{l}v_{1 i} \\
\%\end{array}$} \\
\hline & 0 & 1 & 2 & \begin{tabular}{|l}
3 \\
\end{tabular} & 4 & 5 & & & & & \\
\hline 0.20 & 2506.6 & 1979.1 & 1226.6 & 904.9 & 1418.9 & 494.0 & 2455.1 & 1.8 & 0.02692 & 7.7 & 19.3 \\
\hline 0.25 & 2308.0 & 2049.5 & 1258.3 & 1065.1 & 1264.2 & 1026.1 & 2130.0 & 14.8 & 0.01620 & 35.2 & 13.8 \\
\hline 0.30 & 2929.4 & 1820.7 & 2092.1 & 1442.3 & 382.7 & 930.6 & 2890.2 & 15.6 & 0.03081 & 23.3 & 22.4 \\
\hline 0.35 & 3022.5 & 2653.0 & 1691.9 & 1829.5 & 599.7 & 1321.4 & 3019.4 & 20.8 & 0.02434 & 2.6 & 18.7 \\
\hline 0.40 & 2568.6 & 2325.1 & 2299.2 & 1408.3 & 1739.7 & 442.6 & 3239.2 & 29.6 & 0.02901 & 16.0 & 26.1 \\
\hline 0.45 & 1921.4 & 1758.0 & 1218.3 & 1330.7 & 773.4 & 862.8 & 1957.3 & 21.7 & 0.01822 & 27.1 & 10.4 \\
\hline 0.50 & 2785.1 & 2430.2 & 1987.5 & 1040.2 & 770.4 & 108.4 & 4382.8 & 75.3 & 0.05807 & 132.3 & 47.6 \\
\hline 0.55 & 2323.1 & 2001.1 & 2160.8 & 1123.4 & 1048.3 & 595.2 & 2709.9 & 8.4 & 0.02686 & 7.5 & 19.1 \\
\hline 0.60 & 2837.1 & 1383.7 & 1367.5 & 955.1 & 691.1 & 992.8 & 2136.2 & 14.6 & 0.02198 & 12.1 & 25.6 \\
\hline 0.65 & 3387.0 & 2232.5 & 1684.9 & 1009.8 & 1333.6 & 902.7 & 2932.6 & 17.3 & 0.02477 & 0.9 & 15.2 \\
\hline 0.70 & 2094.5 & 2150.9 & 1919.4 & 900.1 & 770.4 & 375.7 & 2781.9 & 11.3 & 0.03551 & 42.0 & 27.3 \\
\hline & 2607.6 & 2071.3 & 1718.8 & 1182.7 & 981.1 & 732.0 & 2676.9 & 7.1 & 0.02562 & 2.5 & 3.9 \\
\hline
\end{tabular}

Table 4. Virtual experimental results on reconstruction of the dependence between the matrix permeability $k_{2}$ and stresses.

\begin{tabular}{|c|c|c|c|c|c|c|c|c|c|c|c|}
\hline 1 & 2 & 3 & 4 & 5 & 6 & 7 & 8 & 9 & 10 & 11 & 12 \\
\hline \multirow{2}{*}{$\begin{array}{c}P_{i} \\
M P a\end{array}$} & \multicolumn{6}{|c|}{$\sigma_{n}, M P a$} & \multirow{2}{*}{$\begin{array}{c}K_{2} \\
m D\end{array}$} & \multirow{2}{*}{$\begin{array}{l}\theta_{2} \\
\%\end{array}$} & \multirow{2}{*}{$\begin{array}{c}\alpha_{2} \\
M P a^{-1}\end{array}$} & \multirow{2}{*}{$\begin{array}{l}\theta_{\alpha} \\
\%\end{array}$} & \multirow{2}{*}{$\begin{array}{l}v_{2 i} \\
\%\end{array}$} \\
\hline & 0 & 1 & 2 & 3 & 4 & 5 & & & & & \\
\hline 0.20 & 124.9 & 119.9 & 115.1 & 107.4 & 102.5 & 97.0 & 125.9 & 0.7 & 0.00515 & 3.0 & 0.7 \\
\hline 0.25 & 123.1 & 120.8 & 113.2 & 106.1 & 103.2 & 97.3 & 124.6 & 0.4 & 0.00489 & 2.2 & 1.1 \\
\hline 0.30 & 124.8 & 117.7 & 113.7 & 107.2 & 104.0 & 98.0 & 124.3 & 0.6 & 0.00469 & 6.2 & 0.7 \\
\hline 0.35 & 123.6 & 119.3 & 114.8 & 108.4 & 103.6 & 99.2 & 124.5 & 0.4 & 0.00452 & 9.5 & 0.5 \\
\hline 0.40 & 124.3 & 118.9 & 112.1 & 108.1 & 101.0 & 99.1 & 124.1 & 0.8 & 0.00473 & 5.3 & 0.9 \\
\hline 0.45 & 125.0 & 118.9 & 112.8 & 107.1 & 101.8 & 96.6 & 125.0 & 0.0 & 0.00516 & 3.2 & 0.1 \\
\hline 0.50 & 124.8 & 119.0 & 112.2 & 108.8 & 101.8 & 98.0 & 124.7 & 0.2 & $\mid 0.00489$ & 2.2 & 0.6 \\
\hline 0.55 & 123.6 & 117.7 & 114.3 & 107.6 & 101.7 & 97.3 & 124.1 & 0.7 & 0.00484 & 3.2 & 0.7 \\
\hline 0.60 & 124.4 & 119.9 & 112.6 & 105.9 & 102.7 & 96.9 & 124.9 & 0.1 & 0.00508 & 1.6 & 0.7 \\
\hline 0.65 & 126.2 & 119.2 & 112.5 & 107.5 & 101.9 & 96.2 & 125.9 & 0.7 & 0.00534 & 6.9 & 0.3 \\
\hline 0.70 & 123.9 & 118.2 & 114.1 & 109.2 & 102.0 & 97.6 & 124.5 & 0.4 & 0.00480 & 4.1 & 0.7 \\
\hline & 124.4 & 119.0 & 113.4 & 107.6 & 102.4 & 97.6 & 124.8 & 0.2 & 0.00492 & 1.6 & 0.2 \\
\hline
\end{tabular}

At each $P_{i}$, by the least square method, we find the values of $K_{m}$ (columns 8 , Tables 3 and 4) and $\alpha_{m}$ (columns 10) in the empirical relations

$$
k_{m}(i, n) \cong K_{m} \exp \left(-\alpha_{m} \sigma\right),
$$

as well as the relative errors $\theta_{m}=\left|1-K_{m} / K_{m}^{*}\right|$ and $\theta_{\alpha}=\left|1-\alpha_{m} / \alpha_{m}^{*}\right|$ (columns 9 and 11). Columns 12 (Tables 4 and 5) present the variation factors

$$
v_{m i}=\sqrt{N \sum_{n=1}^{N}\left[k_{m}(i, n)-K_{m} \exp \left(-\alpha_{m} \sigma_{n}\right)\right]^{2}} / \sum_{n=1}^{N} k_{m}(i, n)
$$

characterizing the integral approximation error (18), $N=6$ is the number of stress levels in the virtual model experimentation. It is seen that, despite a small error of the input data, at some $P_{i}$ the function $k_{1}(\sigma)$ (line 3 in Fig. 4) is determined at the considerable error as compared with reference function (line 1). After averaging the inversion data of $k_{m}(i, n)$ 
obtained at all values of the input pressure $P_{i}$ (last lines in Tables 4 and 5), the variation factor is never higher than $5 \%$ at any random values $\omega_{s}(i, n)$ in (14) (line 2 in Fig. 4).

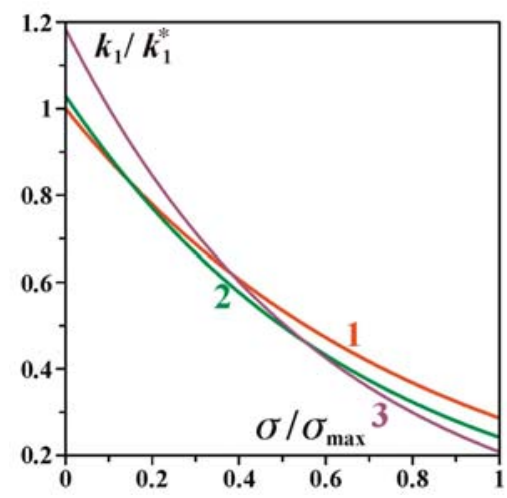

Fig. 4. Stress-dependent permeability $k_{1}$ of fissures.

\section{Conclusion}

The authors have developed and theoretically, based on virtual experimentation, substantiated the method that makes it possible to quantitatively estimate the mass exchange factor $\beta$ and to establish the empirical dependence of permeability on stresses in fractured porous reservoir rocks. The interpretation of the synthetic input data is implemented within the model of continuum with dual porosity, for which the exact solution of the problem on steady-state fluid flow is obtained. The analytical expressions are derived for determining permeabilities of fissures and matrix as well as the coefficient $\beta$ by flow rates measured in three different measurement designs. As yet the value of $\beta$ has been determined only in an operating well by the pressure build-up curve while the proposed procedure allows such estimating in laboratory using standard equipment.

The work of was carried out with partial support of the Russian Foundation for Basic Research (Project No. 18-05-00830) and Program of Federal Scientific Investigations (Identification Number AAAAA-017-117122090002-5).

\section{References}

1. L. P. Dake, The Practice of Reservoir Engineering (Elsevier, 2001)

2. P. R. Robinson, C. S. Hsu, Introduction to Petroleum Technology. Handbook of Petroleum Technology (Springer, 2017)

3. S. N. Zakirov, Gas, Gas-Condensate and Oil-Gas-Condensate Reservoir Engineering. (Struna, 1998)

4. V. L. Karnaukhov, E. M. Piyankova, Modern Methods of Drill-Hole Hydrodynamic Survey (Infra-Inzheneriya, 2010)

5. V. N. Dakhnov, Interpretation of Geophysical Logging Data (Nedra, 1982)

6. T. Djebbar, E. C. Donaldson, Petrophysics. Theory and Practice of Measuring Reservoir Rock and Fluid Transport Properties (Elsevier, 2016)

7. T. V. Dorofeeva, Petroleum reservoirs of Bazhenov formation (Western Siberia). Nedra, (1983).

8. G. I. Barenblatt, Yu. P. Zheltov, I. N. Kochina, Prikl. Mat. Mekh. 24, 5: 852-864 (1960)

9. R. Hill, J. Mech. Phys.Solids. 11, 5: 357-372 (1963) 
10. O. Coussy, Mechanics and Physics of Porous Solids (John Wiley \& Son Ltd, 2010)

11. Y.-S. Wu, Multiphase fluid flow in porous and fractured reservoirs (Elsevier, 2016)

12. S. Salimzadeh, N. Khalili, Int. J. Geomech. 16, 3 (2015)

13. J. Rutqvist, Y.-S. Wu, C.-F.Tsang, G. Bodvarsson, Int. J. of Rock Mech. Min. Sci. 39: 429-442 (2002)

14. J. Douglas jr., T. Arbogast, Dynamics of Fluids in Hierarchical Porous Media, J. H. Cushman, ed. 177-221 (Academic Press, 1990)

15. R.-S. Nie, Y.-F. Meng, Y.-Lu. Jia et al., Transport in Porous Media. 92, 1: 213-235 (2012)

16. M. Presho, S. Wo, V. Ginting, J. of Petroleum Sci. Eng. 77, 3-4: 326-337 (2011)

17. L. A. Nazarova, L. A. Nazarov, J. of Min. Sci. 52, 3: 424-431 (2016)

18. P. Thararoopa, Z. T. Karpyn, T. Ertekinc, J. of Natural Gas Sci. Eng. 8: 121-131 (2012)

19. D. N. Espinoza, M. Vandamme, P. Dangla, J.-M. Pereira, S. Vidal-Gilbert, J. Geophys. Res. Solid Earth. 118: 6113-6123 (2013)

20. L. A. Nazarova, L. A. Nazarov, M. Vandamme, J.-M. Pereira, J. Applied and Industrial Mathematics. 11, 2: 236-243 (2017).

21. L. A. Nazarova, L. A. Nazarov, M. Vandamme, J.-M. Pereira, Doklady Phys. 62, 6: 323-327 (2017)

22. L. J. Hosking, H. R. Thomas, M. Sedighi, Canadian Geotech. J. 55, 6: 839-851 (2018)

23. T. Babadagli, X. Ren, K. Develi, Int. J. Multiph. Flow. 68: 40-58 (2015)

24. B. Jones, L. Brouwers, M. Dippenaar, Hydrogeol. J. 26: 945-961 (2017)

25. T. van Golf-Racht, Fundamentals of Fractured Reservoir Engineering (Elsevier, 1982)

26. F. Kuchuk, J. of Petroleum Technology. 47, 1: 36-41 (1995)

27. D. Bourdet, Handbook of Petroleum Exploration and Production. V.3. Well Test Analysis (Elsevier, 2002)

28. X. Yang, T. J. Lu, T. Kim, Phys Lett. A. 378: 2308-2311 (2014)

29. L. J. Durlofsky, Water Resources Res. 27, 5: 699-708 (1991)

30. Z. Q. Huang, J. Yao, Y. J. Li et al., Commun. Comput. Phys. 9: 180-204 (2011)

31. A. Teimoori, Z. Chen, S. S. Rahman, T. Tran, Petroleum Sci. Tech. 23, 5-6: 693-709 (2005)

32. R. M. Holt, SPE Formation Evaluation. 5: 444-448 (1990)

33. R. Heller, J. Vermylen, M. Zoback, AAPG Bull. 98, 5 : 975-995 (2014)

34. R. Roi, X. Paredes, R. Holtzman, Earth and Planetary Sci.Lett. 493: 198-207 (2018)

35. S. Chabezloo, J. Sulem, S. Guedon, F. Martineau, Int. J. Rock Mech. Min. Sci. 46, 2: 297-306 (2009)

36. Y. Geng, D. Tang, H. Xu et al., Fuel. 202: 12-22 (2017)

37. D. W. Rhett, L. W. Teufel, SPE Paper 24756. SPE Annual Tech. Conf. Exhibition. (1992).

38. M. S. Randall, M. Conway, G. Salter, S. Miller, AAPG Search and Discovery Article N 9012.2011 (2010).

39. H. Daigle, E. Rasromani, K. E. Gray, J. Petr. Sci. Eng. 157: 302-311 (2017)

40. L. A. Nazarova, L. A. Nazarov, M. I. Epov, I. N. Eltsov, J. Min. Sci. 49, 5: 704-714 (2013)

41. I. N. Yeltsov, G. V. Nesterova, A. Y. Sobolev, M. I. Epov, L. A. Nazarova, L. A. Nazarov, Russian Geol. Geoph. 55, 5-6: 775-783 (2014)

42. M. D. Zoback, A. Nur, AAPG Bull. 95: 154-158 (1975)

43. J. Ma, J. Rock Mech. Geotech. Eng. 7, 3: 351-357 (2015)

44. P. Pollard, AIME. 216: 38-43 (1959)

45. RF State Standard 26450.2-85. Rocks. Method for determination of absolute gas permeability coefficient by stationary and non-stationary filtration (1985)

46. www.flowcontrolnetwork.com 